\title{
REPORTE DE CASO
}

\author{
Enfermedad de Crohn ileal. Reporte de un caso \\ lleal Crohn's Disease. A case report \\ Ortiz Villalba $J^{1}$, Macchi Salim $\mathrm{H}^{1}$, Di Martino Ortiz $\mathrm{B}^{2}$ \\ ${ }^{1}$ Profesor Adjunto. Departamento de Gastroenterología y Endoscopia Digestiva. Hospital de Clínicas. \\ Facultad de Ciencias Médicas. ${ }^{2}$ Profesor Asistente. Cátedra de Dermatología. \\ Hospital de Clínicas. Facultad de Ciencias Médicas. San Lorenzo - Paraguay.
}

\section{RESUMEN}

El término Enfermedad Inflamatoria Intestinal (EII) incluye a un grupo de entidades clínicas que se caracterizan por ser procesos inflamatorios crónicos de etiología desconocida, y afectar primordialmente, aunque no de forma exclusiva, al intestino. Actualmente se reconocen varias entidades que se engloban en este concepto: Colitis Ulcerosa (CU), Enfermedad de Crohn (EC), Colitis Inclasificada ( $\mathrm{Cl}$ ), Colitis Microscópica (CM) y Reservoritis (Pouchitis). La Enfermedad de Crohn puede afectar a cualquier tramo del intestino, siendo la localización más frecuente la lleal y Colónica en forma simultánea. El proceso inflamatorio en la EC es transmural y sus síntomas fundamentales son la diarrea generalmente crónica y/o el dolor abdominal. Presentamos el caso de una mujer joven con una EC que compromete exclusivamente al ileon terminal, en la que se realizó el diagnóstico de EC ileal por la clínica, la endoscopía y la anatomía patológica, que respondió satisfactoriamente a la terapéutica instaurada, y se revisa el concepto de la EC con énfasis en su diagnóstico.

Palabras clave: Enfermedad Inflamatoria Intestinal, Enfermedad de Crohn, Ileitis.

Autor correspondiente: Prof. Dra. Beatriz Di Martino Ortiz. Perú 1019 casi Artigas. CP: 1412. Email: beatrizdimartino@gmail.com. Asunción-Paraguay. 


\section{ABSTRACT}

The term Inflammatory Bowel Disease (IBD) includes a group of clinical entities that are characterized by chronic inflammatory processes of unknown etiology, and primarily, but not exclusively, affecting the intestine. Currently, several entities are included in this concept: Ulcerative Colitis (UC), Crohn's Disease (CD), Unclassified Colitis (UC), Microscopic Colitis (CM) and Reservoritis (Pouchitis). Crohn's Disease can affect any part of the intestine, with the most frequent location being ileal and colonic simultaneously. The inflammatory process in CD is transmural and its fundamental symptoms are usually chronic diarrhea and/or abdominal pain. We present the case of a young woman with a CD that exclusively compromises the terminal lleon, in whom the diagnosis of ileal CD was performed with the clinical data, the endoscopic procedure and the pathological study, who responded satisfactorily to the established therapy, and the concept of CD with an emphasis on its diagnosis is reviewed.

Key Words: inflammatory bowel disease, Crohn's disease, ileitis.

\section{INTRODUCCION}

Si bien en nuestro medio la CU es la entidad más frecuente de las Ell, la incidencia de la EC ha ascendido de forma particularmente importante en los últimos años (1-3). Estas enfermedades suelen debutar en la juventud, sobre todo entre los 15 y los 30 años, pero se observan casos nuevos a cualquier edad y, Ilama la atención su incidencia en la infancia.

La prevalencia de EC parece ser mayor en áreas urbanas que en las zonas rurales y también mayor en las clases socio-económicas más altas. La mayoría de los estudios muestran que la incidencia primero comienza a aumentar, entre los de clase social más alta, pero la enfermedad se vuelve más omnipresente con el tiempo.

Sobre todo en la EC existe evidencia de que diversas alteraciones genéticas puntuales influyen no sólo en su aparición, sino además en su fenotipo clínico y, en otras variables como la respuesta a determinados tratamientos.

\section{MATERIALES Y METODOS}

Mujer de 27 años, sin antecedentes quirúrgicos previos, no hipertensa, diabética ni alérgica, y sin antecedentes familiares de carcinoma digestivo o enfermedad celíaca, que consulta por dolor abdominal intermitente previo a las deposiciones y en general cede con las mismas. La localización del dolor es periumbilical, en ocasiones en fosa ilíaca derecha (FID) y flanco derecho (FD). El cuadro se acompaña de episodios de diarrea líquida sin gleras ni sangre, entre dos y seis evacuaciones por día, de aproximadamente dos meses de evolución. Refiere además pérdida de peso de cinco kilogramos desde el inicio del cuadro, meteorismo importante y dispepsia. Niega episodios febriles. No se presentan trastornos urinarios ni ginecológicos.

Recibió tratamiento sintomático para la diarrea y un ciclo de antibióticos con ciprofloxacina 500 mgr dos veces/día, por una semana. 
Por la persistencia de los síntomas consultó y, al examen físico general se constató una paciente lúcida, tranquila, no ictérica y sin signos de anemia, con signos vitales dentro de los límites normales.

Examen físico gastroenterológico: abdomen meteorizado. Dolor discreto en FID y FD. Resto del examen sin particularidades. Ruidos hidroaereos normales.

Auxiliares del diagnóstico:

- Laboratorio: Hemoglobina: 11,8 g/l; Hematocrito: 33\%; GB: 9.800 cél/mm3; N: 60\%; L: 35\%; E: $3 \%$. VSG: $38 \mathrm{~mm} / 1^{\mathrm{a}}$ hora. PCR: 12.

- Bioquímica sanguínea: urea, creatinina, glucemia: normales

- Perfil hepático: normal.

- Lipidograma: normal.

- Anti transglutaminasa y antiendomisio: normales.

- Parámetros tiroideos (TSH, T3 y T4): normales.

- p-ANCA y p-ASCA: dentro de límites normales.

- Orina: normal.

- Frotis de heces: leucocitos: 10-12; hematíes: 20-25; no parásitos. C. difficile: negativo.

- Ecografía abdominal: normal.

Con el diagnóstico de síndrome diarreico crónico de etiología a determinar, a continuación se indicó:

1. Video endoscopía digestiva alta con sedación. Los hallazgos son los siguientes:

- Esófago: mucosa, calibre y motilidad normales.

- Unión Esógafo-gástrica: a $38 \mathrm{~cm}$ de la arcada dentaria superior. No se objetiva endoscópicamente hernia hiatal ni signos de esofagitis por reflujo.

- Estómago: líquido claro en escasa cantidad en lago gástrico. Mucosa de fornix, cuerpo y antro de aspecto endoscópico normal. Pliegues y peristaltismo normales. Región subcardial en retrovisión normal. Se toman biopsias de antro y cuerpo para descartar gastritis e investigar la presencia de Helicobacter pylori.

- Píloro: normal.

- Duodeno: bulbo y D2 con mucosa y pliegues normales. Papila mayor normal.

- Vellosidades normales. Se toman biopsias del bulbo y D2 para investigar Enfermedad Celiaca.

Conclusión: videoendoscopía digestiva alta normal.

La anatomía patológica descarta Enfermedad Celiaca.

2. Videocolonoscopia:

- En el examen anal en retrovisión se constatan hemorroides internas grado I no complicadas. Recto con mucosa, calibre, distribución vascular submucosa y distensibilidad normales. Buena preparación colónica. Se progresa por el colon hasta ciego. Mucosa visualizada, calibre, distribución vascular submucosa y distensibilidad colónica normales. Ciego y valvula ileocecal normales. Fondo cecal normal. 
- Se franquea la válvula ileocecal y se explora $10 \mathrm{~cm}$ de ileon terminal. En ileon terminal, aproximadamente a 7-8 cm de la válvula ileocecal, se visualiza una lesión ulcerosa profunda, fondo con fibrina, bordes sobrelevados de aproximadamente $2-2,5 \mathrm{~cm}$ de tamaño. Mucosa vecina a la úlcera de aspecto normal. Vellosidades normales (Figura 1 y Figura 2). Se toman varias biopsias de la lesión y de la mucosa ileal así como biopsias del colon para descartar colitis microscópica.
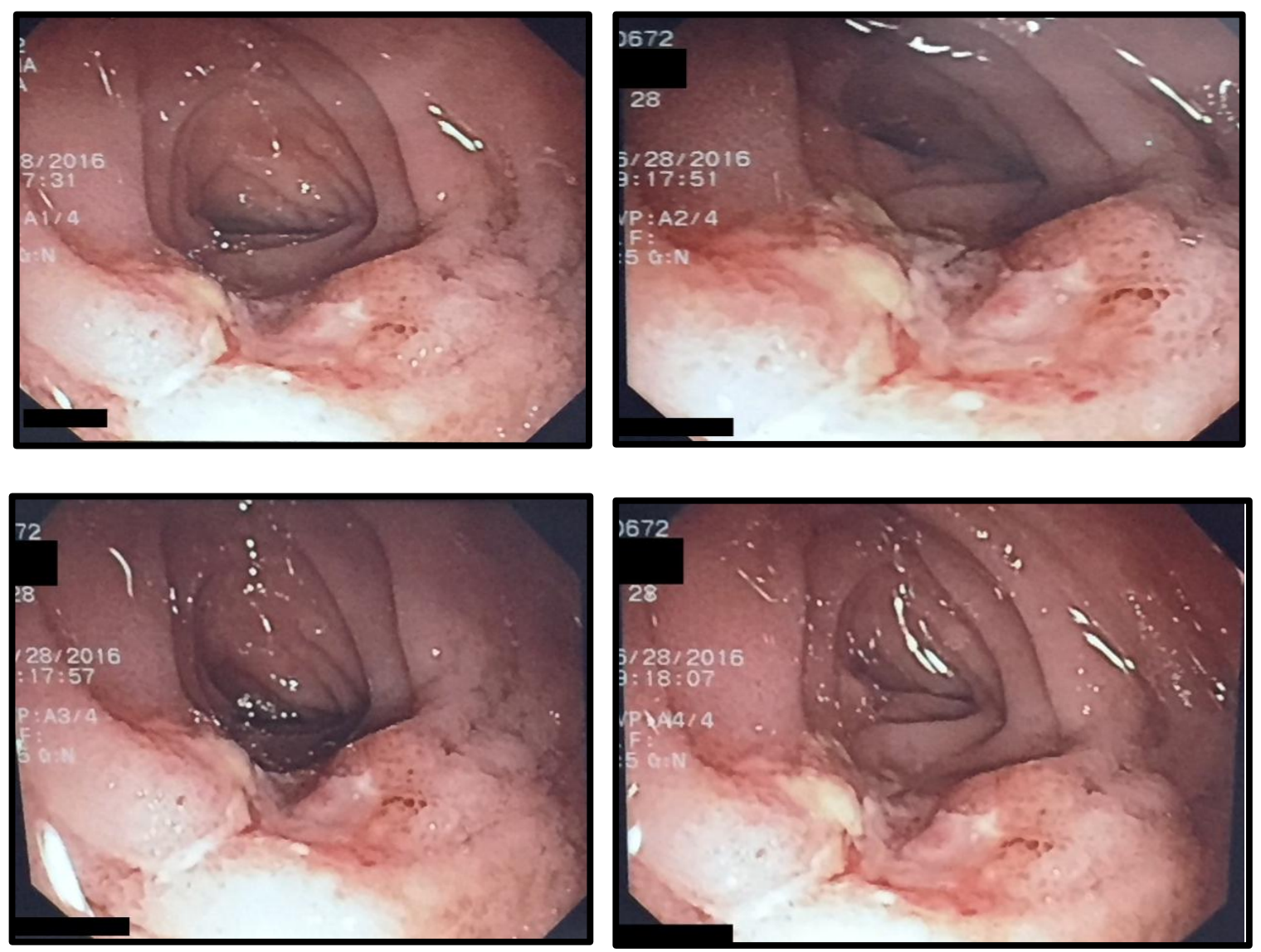

Figura 1: Ulcera aislada profunda en ileon terminal, bordes sobreelevados, fondo con fibrina, tamaño aproximado de $2,5-3 \mathrm{~cm}$. La mucosa vecina circundante es de aspecto normal.

Conclusión: lesión ulcerosa en ileon terminal de etiología a determinar: Enfermedad de Crohn/Tuberculosis/Linfoma.

Anatomía patológica: granulomatosis de la mucosa del ileon terminal, sin necrosis ni supuración, con células gigantes multinucleadas. Tomas negativas para BAAR (Zielh Neelsen). Imagen corresponde a una Enteritis de Crohn. Mucosa colónica normal. Mucosa gástrica y duodenal sin alteraciones significativas (Figura 3). 


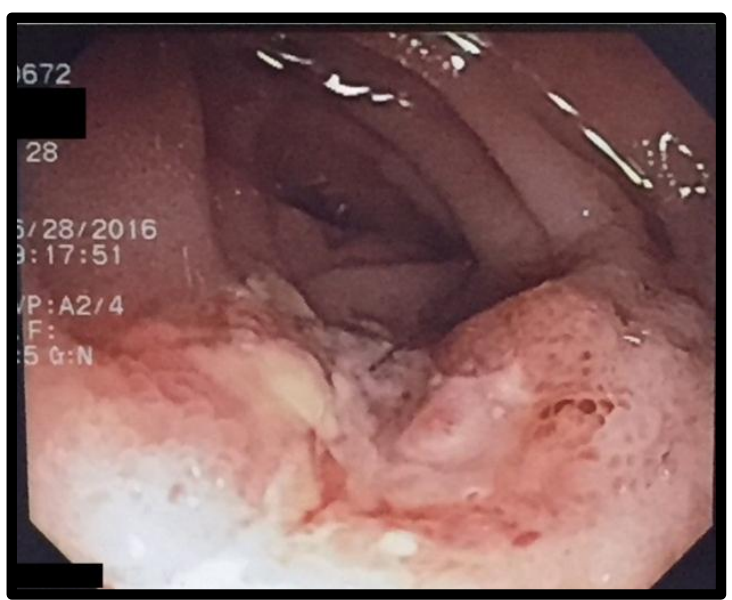

Figura 2: A mayor aumento
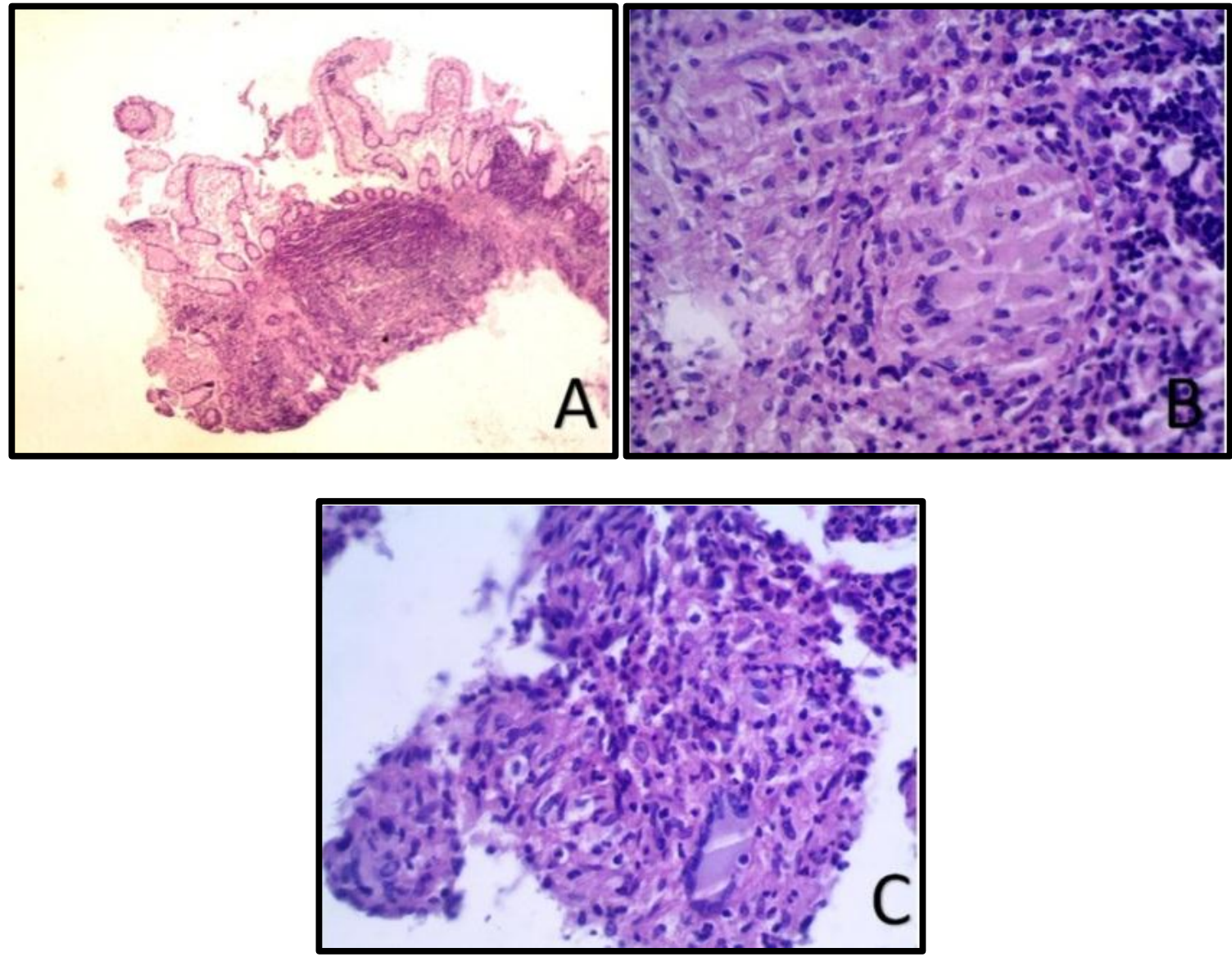

Figura 3: Histopatología. A. Vista panorámica de la mucosa ileal donde se observan pliegues preservados y denso proceso inflamatorio subyacente (HE4X). B. La inflamación está constituida por granulomas de tipo sarcoidal, desnudos, con abundantes células epitelioides centrales. Hay corona linfocitaria periférica escasa (HE40X). C. En algunos granulomas se identifican células gigantes multinucleadas (HE 20X). Ausencia de necrosis o supuración central. 
Con los datos clínicos, endoscópicos e histopatológicos se realiza el diagnóstico final de ENFERMEDAD DE CROHN DE LOCALIZACIÓN ILEAL.

Tratamiento y evolución: Se indica budesonide $9 \mathrm{mg} /$ día por dos meses, con buena evolución clínica, con una y en ocasiones dos deposiciones por día de consistencia sólida y, sin dolor abdominal.

\section{DISCUSION}

La Enfermedad Inflamatoria Intestinal (EII) son un grupo de afecciones intestinales inflamatorias crónicas idiopáticas (1). Las dos categorías principales de enfermedad, Colitis Ulcerosa (CU) y Enfermedad de Crohn (EC), tienen características clínicas y patológicas superpuestas y diferentes. La patogenia de la Ell no está completamente entendida. Factores genéticos y ambientales tales como bacterias luminales intestinales alteradas y mayor permeabilidad intestinal juegan un papel en la desregulación de la inmunidad intestinal, conduciendo a lesiones gastrointestinales.

La Enfermedad de Crohn puede afectar a cualquier tramo del intestino, aunque su localización más frecuente es la íleo-cólica en forma simultánea. En este caso se trata de una enfermedad que afecta exclusivamente al ileon terminal. El proceso inflamatorio es transmural y sus síntomas fundamentales son la diarrea crónica y/o el dolor abdominal. También puede manifestarse por complicaciones como estenosis intestinal y fístulas.

Su clasificación debe realizarse en orden a su localización (ileocólica, ileal, cólica, perianal, o de otras localizaciones), de su patrón clínico (inflamatorio, estenosante o fistulizante), y de su gravedad (leve, moderada, grave). La clasificación de Montreal (5) se ha generalizado y aceptado en los consensos internacionales (2) (Tabla 1).

\begin{tabular}{|l|l|}
\hline Edad al diagnóstico (A) & $\begin{array}{l}\text { A1. } 16 \text { años o menos } \\
\text { A2. 17-40 años } \\
\text { A3. más de } 40 \text { años }\end{array}$ \\
\hline Localización (L) & L1. ileon terminal \\
& L2. colon \\
& L3. ileo cólica \\
& L4. tracto digestivo alto \\
& L1+L4. ileon terminal + tracto digestivo alto \\
& L2+L4. colon + tracto digestivo alto \\
& L3+L4. ileo cólica + tracto digestivo alto \\
\hline Patrón clínico (B) & B1. no estenosante, no fistulizante* o inflamatorio \\
& B1p. inflamatorio con afección perianal asociada \\
& B2. estenosante \\
B2p. estenosante con afección perianal asociada \\
B3. fistulizante \\
* Utilizamos el término fistulizante, aunque el original es penetrante. Este patrón de \\
comportamiento clínico se considera transitorio, porque la historia natural lleva casi \\
siempre a los patrones B2 o B3, o mixtos.
\end{tabular}

Tabla 1. Clasificación de Montreal de la EC ${ }^{5}$ 


\section{En cuanto a la sintomatología, la podemos clasificar en:}

- Relacionadas al daño del tracto digestivo: Diarrea: heces pueden contener moco o sangre. Diarrea nocturna. Incontinencia. Estreñimiento. No paso de gases (en casos de obstrucción del intestino). Dolor o sangrado rectal con movimiento intestinal. Defecación urgente. Tenesmo. Calambres abdominales y dolor en el cuadrante inferior derecho del abdomen común en la EC, o alrededor del ombligo, en el cuadrante inferior izquierdo de moderado a severo en la CU. Náuseas y vómitos pueden ocurrir, pero más en EC que en CU.

- Síntomas generales: fiebre, pérdida de apetito, pérdida de peso, fatiga, sudoración nocturna, retraso del crecimiento, amenorrea primaria.

- Manifestaciones extraintestinales: afecciones musculoesqueléticas, oculares, cutáneas y hepatobiliares.

El diagnóstico se basa en una historia y examen físicos completos, estudios laboratoriales de heces que incluyan cultivos para eliminar causas bacterianas, virales o parasitarias de diarrea, prueba de Clostridium difficile (debe considerarse incluso en ausencia de antecedentes de antibióticos), sangre oculta o leucocitos fecales, lactoferrina, a1-antitripsina, etc.; analítica sanguínea completa, p-ANCA, p-ASCA, estudios para descartar enfermedad celíaca, etc., estudios imagenológicos y la endoscopía digestiva con tomas de biopsias para anatomía patológica. En este caso el examen endoscópico y fundamentalmente la exploración del ileon terminal permitió el diagnóstico. Debemos resaltar que la colonoscopia normal en un paciente con diarrea crónica siempre debe incluir la ileoscopia distal.

En cuanto a la histopatología se evaluará la presencia de granulomas no caseificantes, lo cual sería sugestivo de Enfermedad de Crohn. Granulomas grandes o necróticos, con caseosis deben alertar al médico del diagnóstico de Tuberculosis (TB), especialmente en las regiones en que la TB es endémica. Hay que recordar que la TB puede demostrar en los estudios histológicos una granulomatosis no necrotizante, por lo que como en este caso, se deben realizar las coloraciones especiales (Ziehl Neelsen-ZN) en busca de BAAR. En nuestro caso los granulomas eran pequeños, sarcoideos, no necrotizantes y la coloración de $\mathrm{ZN}$ fue negativa.

El diagnóstico diferencial de la EC incluye a la TB intestinal, tal y como se comentó anteriormente, la enfermedad de Behcet, la CU, la enteropatía por AINES, la Enfermedad Celíaca, entre las principales.

En cuanto al tratamiento éste debe ser individualizado, y es muy conveniente establecer en cada caso no sólo un plan a corto, sino también a medio y largo plazo para el tratamiento integral del paciente.

En la EC el tratamiento depende de la extensión, el fenotipo clínico y la gravedad; siendo esencial el abandono del tabaquismo $(3,6,7)$. El resumen del tratamiento se expone en la Tabla 2. 

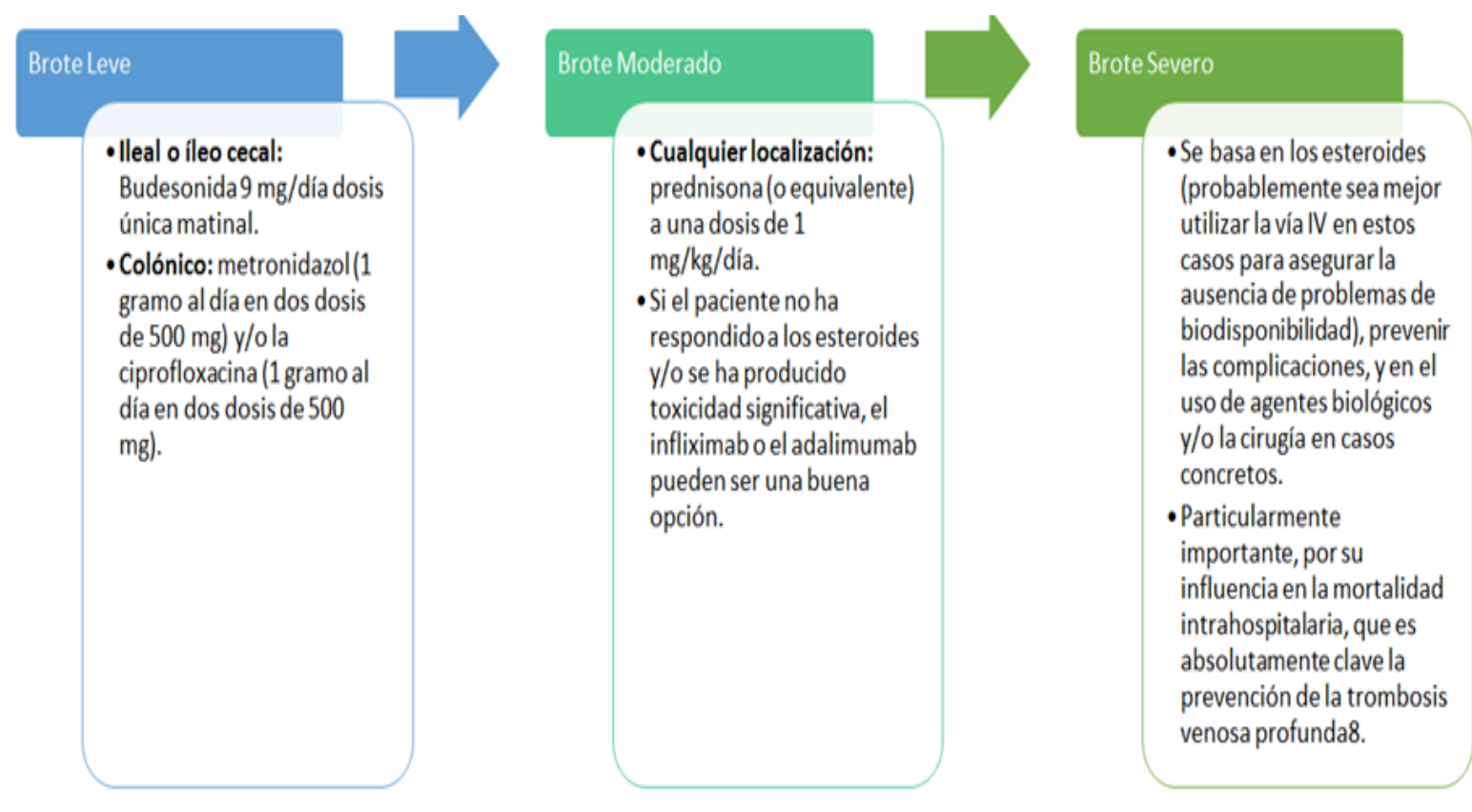

Tabla 2. Algoritmo del tratamiento de la EC

En resumen, nuestro caso reviste interés debido a que se trata de una forma de presentación infrecuente, la ileal exclusiva, con una clínica peculiar en forma de úlcera grande, profunda y aislada. Resaltamos la importancia, en una diarrea crónica, de examinar el íleon terminal aun siendo el colon normal.

\section{REFERENCIAS BIBLIOGRAFICAS}

1. Gassull MA, Gomollón F, Obrador A, Hinojosa J, eds. Enfermedad Inflamatoria Intestinal, 2. ${ }^{a}$ edición. Ergon. Madrid, 2002.

2. Van Assche G, Dignass A, Panés J, Beaugerie L, Karagiannis J, Allez M et al. The second European evidence-based Consensus on the diagnosis and management of Crohn's disease. J Crohns Colitis 2010; 4: 7-27.

3. Dignass A, Van Assche G, Lindsay JO, Lémman M, Söderholm J, Colombel JF et al. The second Eur opean evidence-based Consensus on the diagnosis and management of Crohn's disease. Current $\mathrm{m}$ anagement. J Crohns Colitis 2010; 4: 28-62.

4. Franke A, McGovern DPB, Barrett JC, Wang K, Randford-Smith GL, Ahmad T et al. Genomewide me ta-analysis increases to 71 the number of confirmed Crohn's disease susceptibility loci. Nat Genetics 2010; 42: 1118-1126.

5. Silverberg M, Satsangi J, Ahmad T, Arnott I, Bernstein Ch, Brandt SR et al. Toward and integrated cli nical, molecular, and serological classification of inflammatory bowel disease: report or a working part $y$ of the Montreal World Congress of Gastroenterology. Can J Gastroenterol 2005;19(Suppl A): 5A-36A.

6. Nos P, Clofent J. Enfermedad de Crohn. En Ponce J (editor) Tratamiento de las Enfermedades Gastr oenterológicas. AEG. Elsevier. Barcelona, 2010:293-304.

7. Hinojosa J, Roig JJ. Afectación perianal en la enfermedad de Crohn. En: Ponce J,ed. Tratamiento de las Enfermedades Gastroenterológicas. AEG. Elsevier. Barcelona, 2010:306-318. 